\title{
Microglandular Hyperplasia of the Cervix- A Diagnostic Challenge
}

\section{Sara Maria Bernardes da Cunha ${ }^{1^{*}}$ (i), Ana Marta Pinto ${ }^{2}$, Sofia Raposo ${ }^{3}$, Maria do Carmo Cruz ${ }^{4}$, Rita Sousa ${ }^{3}$, Luís Sá ${ }^{3}$ and Amália Pacheco ${ }^{4}$}

${ }^{1}$ Department of Gyneacology and Obstetrics, Centro Hospitalar de Entre o Douro e Vouga, Santa Maria da Feira, Portugal ${ }^{2}$ Department of Gyneacology and Obstetrics, Centro Hospitalar Baixo Vouga, Aveiro, Portugal

${ }^{3}$ Department of Gyneacology, Instituto Português de Oncologia, Coimbra, Portugal

${ }^{4}$ Department of Gyneacology and Obstetrics, Centro Hospitalar e Universitário de Faro, Algarve, Portugal

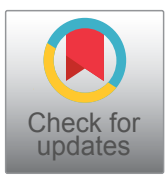

*Corresponding author: Sara Maria Bernardes da Cunha, Department of Gyneacology and Obstetrics, Centro Hospitalar de Entre o Douro e Vouga, R. Dr. Cândido Pinho 5, Santa Maria da Feira, 4520-211, Portugal, Tel: +351-917446965

\begin{abstract}
Microglandular hyperplasia was described for the first time in 1968 and referred to a benign lesion of the cervix, which mimics adenocarcinoma.

The reported incidence is about $15 \%$, leading to insufficient experience in pathological identification. The association with hormonal exposition has been assumed. However, it is not consensual.

We report a clinical case of a 22-years-old women manifesting otorrhagia. In the physical assessment, a suspicious exophytic bulky lesion was observed. The macroscopic appearance suggested malignancy. The first histological sampling was insufficient to rule out malignancy and magnetic resonance suggested a IIA cervical cancer. The clinical anatomopathological discrepancy demand to further study. The patient was forwarded for observation at a tertiary centre, where the clinical-pathological discrepancy led a new cervical biopsy. Specimen was observed for multiple laboratories revelling a clinical challenge of the diagnosis and $\mathrm{MH}$ was suggested. Immuno-histochemical typical expression aided the final diagnostic. Hormonal treatment suspension resulted in clinical improvement.

This report highlights the importance of differential diagnosis with malignancy in case of macroscopical exuberant $\mathrm{MH}$ lesion in a young reproductive-age woman. The awareness of this condition can lead to a better approach in reproductive age woman, as a more conservative treatment, potentially providing a better quality of life.
\end{abstract}

\section{Keywords}

Microglandular hyperplasia, Cervical benign disease, Differential diagnosis

\section{Introduction}

Many benign, proliferative, or reactive processes occur throughout the female genital tract and may mimic benign or malignant tumors.

The term "microglandular hyperplasia" (MH) was described for the first time in 1968 by Kyriakos, et al. $[1,2]$. Young and Scully reported atypical forms of $\mathrm{MH}$ of the cervix which mimics carcinoma [3]. The increasing incidence of malignant and premalignant endocervical glandular lesions can lead to a more challenging differential diagnosis with benign conditions [4].

The incidence described is about $15 \%$, this relative low incidence manifests as a low experience in the identification of this benign lesion [2,5]. $\mathrm{MH}$ as a possible association with long hormone exposition, particularly the progestin component. However this association is not clear [1]. Around 6-10\% of the cases can be found in postmenopausal women, raising confounders regarding this association. Nevertheless, this tumour typically occurs in reproductive-age women [1,2].

It was reported that $13.2 \%$ of patients using a levonorgestrel-releasing intrauterinewhile another study showed $1.3 \%$ correlation between $\mathrm{MH}$ and progestin exposure [6]. It can occur in till $50 \%$ of women without pregnancy history [2]. Greeley, et al. did not find an association of $\mathrm{MH}$ and oral contraception use or in cases of recent pregnancy history [6].

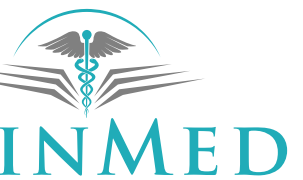

NTERNATIONAL LIBRARY

Citation: Cunha SMB, Pinto AM, Raposo S, Cruz MC, Sousa R, et al. (2022) Microglandular Hyperplasia of the Cervix- A Diagnostic Challenge. Obstet Gynecol Cases Rev 9:217. doi.org/10.23937/23779004/1410217

Accepted: January 20, 2022: Published: January 22, 2022

Copyright: (C) 2022 Cunha SMB, et al. This is an open-access article distributed under the terms of the Creative Commons Attribution License, which permits unrestricted use, distribution, and reproduction in any medium, provided the original author and source are credited. 
Most often $\mathrm{MH}$ is asymptomatic at presentation and found in routine cervical assessment. Due to erosion and proliferation of glandular tissue can also manifest with abnormal vaginal bleeding or discharge $[1,2]$. No statistically significant association between the $\mathrm{MH}$ and squamous dyskaryosis or malignant change is seen [5]. Typically isn't associated with Human papillomavirus (HPV) infection however can be suspected due to cytological smear alterations [6].

In histological analysis $\mathrm{MH}$ is characterized with a tightly packed glands lined by cuboidal to low columnar epithelium with round, uniform, bland nuclei and rare mitoses [4]. Adenocarcinoma can easily be mistaken when architecturally complex, inflamed, or presenting with more unusual solid or trabecular patterns [7]. Inflammatory cells can be present and immunohistochemical markers like p53, CEA, MIBI and Ki67 can be useful for the differential diagnosis if histology is not clear [8].

The clinical relevance of this condition is associated in one hand with the difficulty of the differential diagnosis with invasive adenocarcinoma and in other hand with the low frequency of the lesion leading to a possible lack of experience with this feature $[2,5,6]$.

In our clinical report we highlight the macroscopical exuberant lesion in a young reproductive age woman, focusing on the importance of the malignancy exclusion too ensure a treatment compatible with future reproduction.

\section{Clinical Case}

Patient aged 22 years presented with an extensive cervical lesion. The patient was a non-smoker without relevant past clinical history. She presented with metrorrhagia related to sexual intercourse. The patient had begun her sexual activity in the previous year and had previously completed 3 doses of HPV vaccine. She had been on oral contraceptives. Macroscopically the lesion was consistent with malignant cervical involvement. The first biopsy was inconclusive. A second biopsy was conducted and discussed between centres. Specimen revealed microglandular hyperplasia; however, the clinical-anatomopathological discordance was high, as the extensive lesion pointed to an invasive disease and the histological analyses reported a benign lesion. In the Magnetic resonance imaging, the cervix dimensions were $42 \times 27 \times 23 \mathrm{~mm}$ with a plain morphology extended until the left vaginal recess. No suggestive figures of lymphatic metastasis were identified. Image was suggestive of a IIA cervical carcinoma.

The anatomopathological result was reviewed and sent to a tertiary oncology centre.

On examination, the cervix was enlarged and friable, transformed in exophytic lesions with papillary aspects (Figure 1).

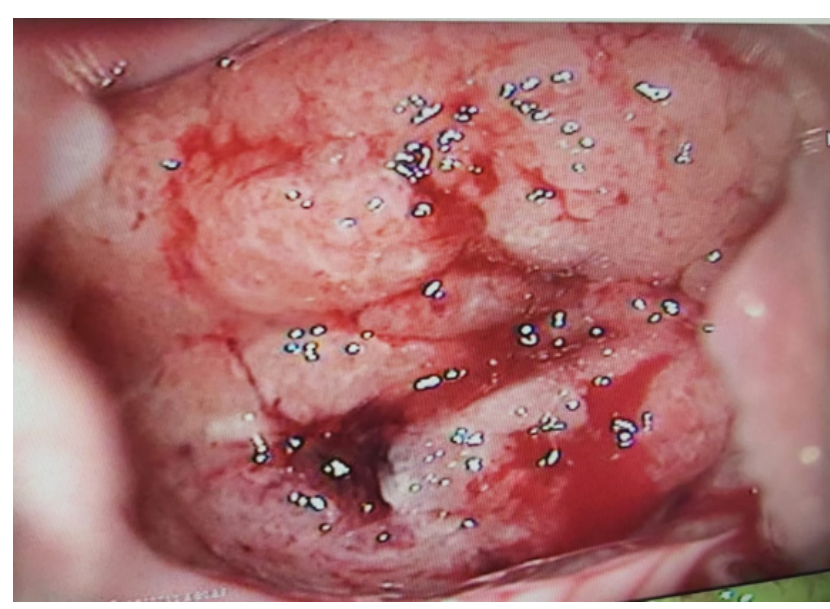

Figure 1: Initial colposcopy appearance of the cervix.

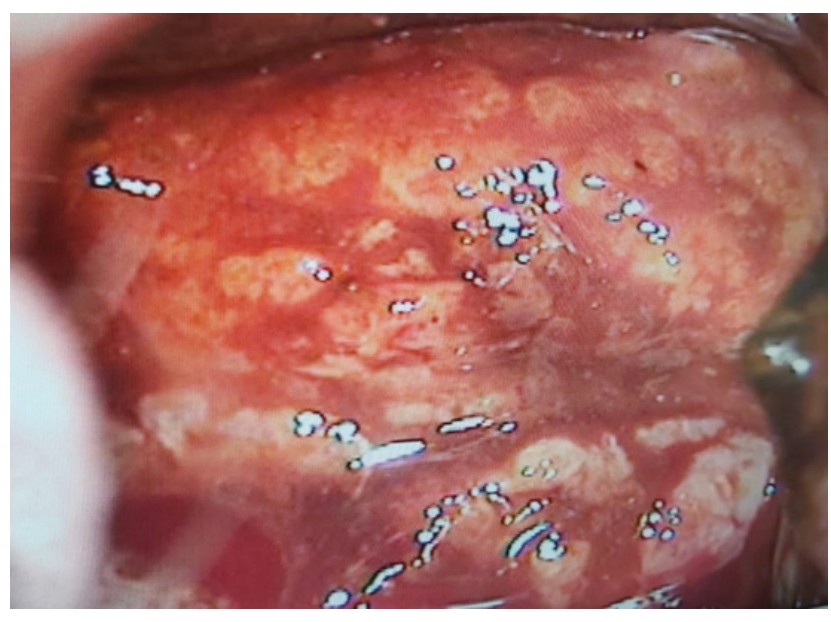

Figure 2: Initial colposcopy appearance of the cervix after acetic acid application.

After the application of acetic acid, a yellow colouration appeared in the lesion (Figure 2).

At bimanual palpation, the lesion was softened and extended to all the cervical area. Parameters were free. A cytological smear with HPV analyses was conducted.

The cytology revelled an atypical squamous cell of undetermined significance, and oncogenic Papillomavirus was negative.

Due to clinical and anatomopathological discrepancy and inability to rule out malignancy, the evaluation with cervical biopsy was repeated.

Histology of the fragment revealed a complex architecture without atypic or mitotic figures and a glandular endocervical proliferation of unknown origin with florid proliferation suggestive of $\mathrm{MH}$. Some neutrophilic infiltration and erosion were also observed. Immunohistochemistry: p16, CEA and p63 negative and positivity to CK7.

A suspension of exogenous hormones was decided. Six months after, we observed a consistent clinical improvement, with lesion regression (Figure 3). We offered non-hormonal contraceptive methods to the 


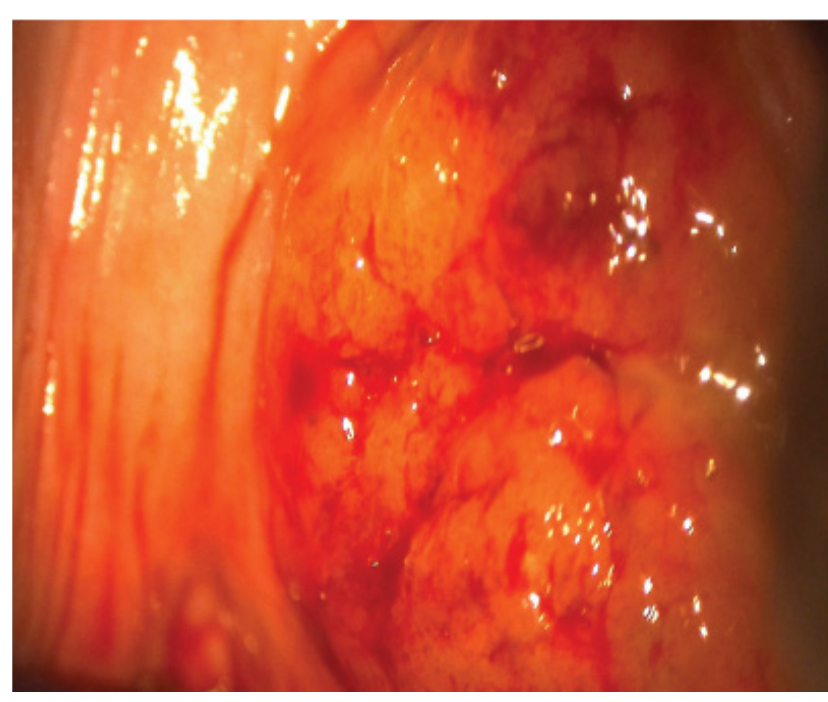

Figure 3: Colposcopy after hormonal suspension.

patient. Due to the macroscopic improvement of the lesion, and benign histology, cervical vaporization was decided and conducted to symptom improvement.

In the 6 months control a lesion relapsed was diagnosed, and a cervical conization was conducted. Anatomopathological specimen report confirmed glandular hyperplasia without neoplastic aspects. In 6 months revaluation cervical macroscopic aspects were normal.

\section{Conclusion}

$\mathrm{MH}$ is a proliferative benign lesion often found in biopsy material, demonstrating as a proliferative reaction of endocervical glands $[9,10]$.

Association with oral contraceptives or hormonal exposition - as in pregnancy, has been proposed and observed in several studies, however, this is not yet consensual, as $\mathrm{MH}$ is present in postmenopausal women cervical biopsies (6.5\%) [9-12]. Young and Scully [11], who found an association with oral contraceptives in $67 \%$ of cases, progestational therapy in $7 \%$ and pure estrogen therapy in $3.5 \%$; it was idiopathic in $22 \%$ of cases $[10,12]$. In this case, the suspension of hormonal exposition led to a regression of the lesion, showing that it plays a role in $\mathrm{MH}$ pathophysiology despite controversial evidence.

The presented clinical case also highlights the importance of differential diagnosis and multidisciplinary approach. It was particularly imperative to distinguish $\mathrm{MH}$ from adenocarcinoma, most importantly, due to the exuberant clinical manifestation and parametric invasion. The histological evaluation is obviously a main aspect of the management, ensuring a benign aetiology, thus supporting the expectant attitude, as opposed to a more radical approach, which could compromise the patient's reproductive potential [9].

Premalignant and malignant endocervical glandular lesions are much more uncommon than their squamous counterparts in the cervix, but are increasing in prevalence [4].

Adenocarcinoma incidence is increasing. $\mathrm{MH}$ is a cause of false positive findings in cervical cytology because of the presence of endocervical glandular atypia [10]. Due to this possible confounder, diagnosis can be challenging specially in young reproductive women, where reproductive potential is an issue, as in our clinical report [11].

$\mathrm{MH}$ is a benign lesion with clinical exuberant manifestation, often mistaken to a possible advanced adenocarcinoma. Is often related to hormonal exposure, particularly birth-control pills. In this case report, we focused on the macroscopic aspect of $\mathrm{MH}$ in a young patient, to whom fertility preservation is a key aspect of the treatment. Despite the ambiguous relation to progestin exposure, this trigger removal resulted in lesion partial regression in this clinical setting which combined with minimal surgical approach resulted in a fertility preservation.

We emphasize the requirement of a histological assessment of suspicious lesions, and the relevance of immunological categorization as a diagnostic tool. The awareness of health-care providers ensures an appropriate diagnostic approach and patient counselling.

\section{Statements}

\section{Acknowledgement}

We certify that this material has not been published previously and is not under consideration by another journal. We further certify that all authors have had substantive involvement in the preparation of this manuscript and are fully familiar with its content.

\section{Disclosure statement}

We have no conflicts of interest to disclose.

\section{Funding sources}

There was no funding assigned to this work.

\section{Author contributions}

Sara Cunha and Ana Pinto were responsible for the conception and drafting of the work. Sara Cunha, Ana Pinto, Sofia Raposo and Rita Sousa were responsible for the acquisition, analysis and interpretation of information contained within the manuscript. Sofia Raposo, Rita Sousa, Luís Sá, Maria do Carmo Cruz and Amália Pacheco contributed for the design of the work, to revise and to approve the final manuscript. All authors approve the final version to be published and ensure that aspects related with the integrity/accuracy are accomplished.

\section{References}

1. Nucci MR (2014) Pseudoneoplastic glandular lesions of the 
uterine cervix: A selective review. Int J Gynecol Pathol 33: 330-338.

2. Harrison BT, Nucci MR (2018) Glandular pseudoneoplasms of the uterine cervix. AJSP: Reviews \& Reports 23: 73-83.

3. Young RH, Scully RE (1989)Atypical forms of microglandular hyperplasia of the cervix simulating carcinoma. A report of five cases and review of the literature. Am J Surg Pathol 13: $50-56$.

4. McCluggage WG (2003) Endocervical glandular lesions: Controversial aspects and ancillary techniques. J Clin Pathol 56: 164-173.

5. Nichols TM, Fidler HK (1971) Microglandular hyperplasia in cervical cone biopsies taken for suspicious and positive cytology. Am J Clin Pathol 56: 424-429.

6. Heller DS, Nguyen L, Goldsmith LT (2016) Association of cervical microglandular hyperplasia with exogenous progestin exposure. Journal of Lower Genital Tract Disease 20: 162-164.
7. Wright TC, Ferenczy A (1994) Benign diseases of the cervix. In: Kurman RJ, Blaustein's pathology of the female genital tract. Springer, New York, 203-227.

8. Montero Macias R, Bats A, Balaya V, Ngo C, Delomenie M, et al. (2019) 347 Endocervical microglandular hiperplasia in a 21 years old patient. International Journal of Gynecologic Cancer 29: A143-A4.

9. Valente PT, Schantz HD, Schultz M (1994) Cytologic atypia associated with microglandular hyperplasia. Diagnostic Cytopathology 10: 326-331.

10. Alvarez-Santin C, Sica A, Rodriguez MC, Feijo A, Garrido $G$ (1999) Microglandular hyperplasia of the uterine cervix. Acta Cytologica 43: 110-113.

11. Medeiros F, Bell DA (2010) Pseudoneoplastic lesions of the female genital tract. Arch Pathol Lab Med 134: 393-403.

12. Padrao IL, Andrade LALDA (2006) Microglandular hyperplasia of the cervix: Frequency in cone specimens, histological patterns, clinical aspects and immunohistochemical markers for differential diagnosis with adenocarcinoma. J Bras Patol Med Lab 42: 219-225. 